\title{
On Holomorphic Artin L-functions
}

\author{
FLORIN NICOLAE \\ Simion Stoilow Institute of Mathematics \\ of the Romanian Academy \\ P.O.BOX 1-764 \\ RO-014700 Bucharest \\ email:florin.nicolae@imar.ro
}

July 19, 2018

\begin{abstract}
Let $K / \mathbb{Q}$ be a finite Galois extension, $s_{0} \in \mathbb{C} \backslash\{1\}, \operatorname{Hol}\left(s_{0}\right)$ the semigroup of Artin L-functions holomorphic at $s_{0}$. If the Galois group is almost monomial then Artin's L-functions are holomorphic at $s_{0}$ if and only if $\operatorname{Hol}\left(s_{0}\right)$ is factorial. This holds also if $s_{0}$ is a zero of an irreducible L-function of dimension $\leq 2$, without any condition on the Galois group.

Key words: Artin L-function; Artin's holomorphy conjecture MSC: 11R42
\end{abstract}

Let $K / \mathbb{Q}$ be a finite Galois extension with the Galois group $G, \chi_{1}, \ldots, \chi_{r}$ the irreducible characters of $G$ with the dimensions $d_{1}:=\chi_{1}(1), \ldots, d_{r}:=$ $\chi_{r}(1), f_{1}=L\left(s, \chi_{1}, K / \mathbb{Q}\right), \ldots, f_{r}=L\left(s, \chi_{r}, K / \mathbb{Q}\right)$ the corresponding Artin L-functions,

$$
A r:=\left\{f_{1}^{k_{1}} \cdot \ldots \cdot f_{r}^{k_{r}} \mid k_{1} \geq 0, \ldots, k_{r} \geq 0\right\}
$$

the multiplicative semigroup of all L-functions. Artin proved that $f_{1}, \ldots, f_{r}$ are multiplicatively independent ([1], Satz 5, P. 106 ), so $A r$ is factorial with the set of primes $\left\{f_{1}, \ldots, f_{r}\right\}$. For $s_{0} \in \mathbb{C}, s_{0} \neq 1$, let $\operatorname{Hol}\left(s_{0}\right)$ be the subsemigroup of $A r$ consisting of the L-functions which are holomorphic at $s_{0}$. Artin conjectures that every L-function is holomorphic at $s_{0}$. This is true for monomial Galois groups. If $G$ is isomorphic to $A_{5}$, the alternating group on five elements, it was proved in ([3], Theorem 3) that the L-functions are 
holomorphic at $s_{0}$ if and only if $\operatorname{Hol}\left(s_{0}\right)$ is factorial. A finite group $G$ is called almost monomial if for every distinct irreducible characters $\chi$ and $\psi$ of $G$ there exist a subgroup $H$ of $G$ and a linear character $\varphi$ of $H$ such that the induced character $\varphi^{G}$ contains $\chi$ and does not contain $\psi$. Every monomial group and every quasi monomial group in the sense of ([2]) are almost monomial. The group $A_{5}$ is almost monomial.

Theorem 1. If the Galois group is almost monomial, then the following assertions are equivalent:

1) Artin's conjecture is true: $\operatorname{Hol}\left(s_{0}\right)=A r$.

2) The semigroup $\operatorname{Hol}\left(s_{0}\right)$ is factorial.

Proof. 1) $\Rightarrow 2$ ): If the L-functions are holomorphic at $s_{0}$, then $\operatorname{Hol}\left(s_{0}\right)=A r$ is factorial.

$2) \Rightarrow 1)$ : Suppose that Artin's conjecture is not true. Then there exists $1 \leq k \leq r$ such that

$$
\operatorname{ord}\left(f_{k}\right)<0,
$$

where ord $(f)$ denotes the order of the Artin L-function $f$ at $s_{0}$. The Dedekind zeta function $\zeta_{K}$ of $K$ decomposes as

$$
\zeta_{K}=f_{1}^{d_{1}} \cdot \ldots \cdot f_{r}^{d_{r}} .
$$

Since $\zeta_{K}$ is holomorphic in $\mathbb{C} \backslash\{1\}$ it holds that

$$
\operatorname{ord}\left(\zeta_{K}\right) \geq 0 \text {. }
$$

From (1), (2) and (3) it follows that there exists $l \in\{1, \ldots, r\}$ such that

$$
\operatorname{ord}\left(f_{l}\right)>0 \text {. }
$$

For $j \in\{1, \ldots, r\}$ let

$$
m_{j}:=\min \left\{m \geq 0: \operatorname{ord}\left(f_{l}^{m} \cdot f_{j}\right) \geq 0\right\} .
$$

Since the L-functions $f_{1}, \ldots, f_{r}$ are multiplicatively independent the elements $f_{l}^{m_{1}} \cdot f_{1}, \ldots, f_{l}^{m_{r}} \cdot f_{r}$ are irreducible in $\operatorname{Hol}\left(s_{0}\right)$. We have seen in [3], p. 2862, that $\operatorname{Hol}\left(s_{0}\right)$ is a positive affine semigroup which generates the free abelian group $\left\{f_{1}^{k_{1}} \cdot \ldots f_{r}^{k_{r}} \mid k_{1} \in \mathbb{Z} 0, \ldots, k_{r} \in \mathbb{Z}\right\}$ with the basis $f_{1}, \ldots, f_{r}$. The Hilbert basis $\mathcal{H}$ of $\operatorname{Hol}\left(s_{0}\right)$ is the uniquely determined minimal system of generators of $\operatorname{Hol}\left(s_{0}\right)$, hence $\operatorname{Hol}\left(s_{0}\right)$ is factorial if and only if $\mathcal{H}$ has $r$ elements. It follows that

$$
\mathcal{H}=\left\{f_{l}^{m_{1}} \cdot f_{1}, \ldots, f_{l}^{m_{r}} \cdot f_{r}\right\} .
$$

From (1) it follows that $m_{k}>0$. Since the Galois group $G$ is almost monomial there exist a subgroup $H$ of $G$ and a linear character $\psi$ of $H$ such that the 
induced character $\psi^{G}$ contains $\chi_{k}$ and does not contain $\chi_{l}$. By classfield theory the Artin L-function $L\left(s, \psi^{G}, K / \mathbb{Q}\right)$ is a Hecke L-function so it is holomorphic at $s_{0}$. Then $L\left(s, \psi^{G}, K / \mathbb{Q}\right)$ is a product of elements of $\mathcal{H}$. Since $\psi^{G}$ contains $\chi_{k}$ the Artin L-function $L\left(s, \psi^{G}, K / \mathbb{Q}\right)$ contains $L\left(s, \chi_{k}, K / \mathbb{Q}\right)=$ $f_{k}$ so it contains $f_{l}^{m_{k}} \cdot f_{k}$ and so $f_{l}$ since $m_{k}>0$. On the other hand, since $\psi^{G}$ does not contain $\chi_{l}$ the Artin L-function $L\left(s, \psi^{G}, K / \mathbb{Q}\right)$ does not contain $L\left(s, \chi_{l}, K / \mathbb{Q}\right)=f_{l}$, a contradiction.

We don't know whether any finite group $G$ is almost monomial. We think that theorem 1 is true without no condition on the Galois group. We can prove only a partial result:

Theorem 2. If $s_{0}$ is a zero of some $f_{l}$ with $d_{l} \leq 2$ then the following assertions are equivalent:

1) Artin's conjecture is true: $\operatorname{Hol}\left(s_{0}\right)=A r$.

2) The semigroup $\operatorname{Hol}\left(s_{0}\right)$ is factorial.

Proof. 1) $\Rightarrow$ 2) is clear.

$2) \Rightarrow 1$ ): Suppose that Artin's conjecture is not true. For $j \in\{1, \ldots, r\}$ let

$$
m_{j}:=\min \left\{m \geq 0: \operatorname{ord}\left(f_{l}^{m} \cdot f_{j}\right) \geq 0\right\} .
$$

As in the proof of theorem 1 we have that the Hilbert basis of $\operatorname{Hol}\left(s_{0}\right)$ is

$$
\mathcal{H}=\left\{f_{l}^{m_{1}} \cdot f_{1}, \ldots, f_{l}^{m_{r}} \cdot f_{r}\right\} .
$$

Since $\zeta_{K} \in \operatorname{Hol}\left(s_{0}\right)$ there exist $a_{1} \geq 0, \ldots, a_{r} \geq 0$ such that

$$
\zeta_{K}=\prod_{j=1}^{r}\left(f_{l}^{m_{j}} \cdot f_{j}\right)^{a_{j}}
$$

so

$$
\begin{gathered}
f_{1}^{d_{1}} \cdot \ldots \cdot f_{r}^{d_{r}}=\prod_{j=1}^{r}\left(f_{l}^{m_{j}} \cdot f_{j}\right)^{a_{j}}, \\
d_{l}=a_{l}+\sum_{j=1, j \neq l}^{r} m_{j} a_{j}, \\
d_{j}=a_{j}, j \neq l
\end{gathered}
$$

since $f_{1}, \ldots, f_{r}$ are multiplicatively independent and $m_{l}=0$. Hence

$$
d_{l}=a_{l}+\sum_{j=1, j \neq l}^{r} m_{j} d_{j} .
$$


Suppose that there exists $k \neq l$ such that

$$
\operatorname{ord}\left(f_{k}\right)>0 .
$$

For $j \in\{1, \ldots, r\}$ let

$$
n_{j}:=\min \left\{m \geq 0: \operatorname{ord}\left(f_{k}^{m} \cdot f_{j}\right) \geq 0\right\} .
$$

It follows that

$$
\mathcal{H}=\left\{f_{k}^{n_{1}} \cdot f_{1}, \ldots, f_{k}^{n_{r}} \cdot f_{r}\right\}
$$

hence

$$
\begin{aligned}
\left\{f_{l}^{m_{1}} \cdot f_{1}, \ldots, f_{l}^{m_{r}} \cdot f_{r}\right\} & =\left\{f_{k}^{n_{1}} \cdot f_{1}, \ldots, f_{k}^{n_{r}} \cdot f_{r}\right\}, \\
f_{l}^{m_{j}} \cdot f_{j} & =f_{k}^{n_{j}} \cdot f_{j}
\end{aligned}
$$

for $j \neq k, l$,

$$
\begin{gathered}
f_{l}^{m_{j}}=f_{k}^{n_{j}}, \\
m_{j}=n_{j}=0, \\
\mathcal{H}=\left\{f_{j}: j \neq k, l\right\} \cup\left\{f_{l}^{m_{k}} \cdot f_{k}, f_{l}\right\}=\left\{f_{j}: j \neq k, l\right\} \cup\left\{f_{k}^{n_{l}} \cdot f_{l}, f_{k}\right\}, \\
f_{k}=f_{l}^{m_{k}} \cdot f_{k}, \\
f_{l}^{m_{k}}=1, \\
m_{k}=0, \\
n_{l}=0, \\
\mathcal{H}=\left\{f_{1}, \ldots, f_{r}\right\} .
\end{gathered}
$$

This means that $f_{1}, \ldots, f_{r}$ are holomorphic at $s_{0}$, so Artin's conjecture is true, a contradiction. It follows that

$$
\operatorname{ord}\left(f_{k}\right) \leq 0 \text { for every } k \neq l .
$$

Since we have supposed that Artin's conjecture is not true there exists $k \neq l$ such that

$$
\operatorname{ord}\left(f_{k}\right)<0 .
$$

Since $m_{j}=0$ if $\operatorname{ord}\left(f_{j}\right)=0$ from (4) and (5) it follows that

$$
d_{l}=a_{l}+\sum_{j: \operatorname{ord}\left(f_{j}\right)<0} m_{j} d_{j},
$$

hence

$$
d_{l} \geq \sum_{j: \operatorname{ord}\left(f_{j}\right)<0} m_{j} d_{j}
$$


since $a_{l} \geq 0$. For any $j$ with $\operatorname{ord}\left(f_{j}\right)<0$ we have that $d_{j} \geq 2$, since if $d_{j}=1$ then by classfield theory the L-function $f_{j}$ is a Hecke L-function so it is holomorphic at $s_{0}$. Since $d_{l} \leq 2$ it follows from (6) that there exists only one $k$ such that $\operatorname{ord}\left(f_{k}\right)<0$. This implies $d_{k}=2, m_{k}=1, d_{l}=2$, $\mathcal{H}=\left\{f_{j}: j \neq k\right\} \cup\left\{f_{l} \cdot f_{k}\right\}$. By a result of Rhoades improving the theorem of Aramata-Brauer ( 4], Theorem 2, p. 359) the function $\zeta_{K} \cdot f_{k}$ is holomorphic in $\mathbb{C} \backslash\{1\}$, so $\zeta_{K} \cdot f_{k} \in \operatorname{Hol}\left(s_{0}\right)$. Then $\zeta_{K} \cdot f_{k}$ is a product of elements of $\mathcal{H}$ : there exist $b_{1}, \ldots, b_{r} \geq 0$ such that

$$
\begin{gathered}
\zeta_{K} \cdot f_{k}=\left(\prod_{j \neq k} f_{j}^{b_{j}}\right) \cdot\left(f_{l} \cdot f_{k}\right)^{b_{k}} \\
\left(\prod_{j \neq k} f_{j}^{d_{j}}\right) \cdot f_{k}^{d_{k}+1}=\left(\prod_{j \neq k} f_{j}^{b_{j}}\right) \cdot\left(f_{l} \cdot f_{k}\right)^{b_{k}} \\
b_{k}=d_{k}+1, d_{l}=b_{l}+b_{k}, \\
d_{l} \geq d_{k}+1=3
\end{gathered}
$$

in contradiction with $d_{l}=2$. 


\section{References}

[1] Artin, E.: Über eine neue Art von L-Reihen. Abh. Math. Sem. Hamburg 3, 89-108 (1924).

[2] Nicolae, F.: Artin's L-functions and one-dimensional characters. J. Number Theory 124, No. 1, 26-30 (2007).

[3] Nicolae, F.: On the semigroup of Artin's $L$-functions holomorphic at $s_{0}$. J. Number Theory 128, No. 11, 2861-2864 (2008).

[4] Rhoades, S. L.: A generalization of the Aramata-Brauer theorem. Proc. Amer. Math. Soc.119, 357-364 (1993) 